\title{
Tacrolimus therapy as an alternative to thiopurines for maintaining remission in patients with refractory ulcerative colitis.
}

\author{
AUTHOR(S): \\ Yamamoto, Shuji; Nakase, Hiroshi; Matsuura, \\ Minoru; Masuda, Satohiro; Inui, Ken-ichi; Chiba, \\ Tsutomu
}

\section{CITATION:}

Yamamoto, Shuji ...[et al]. Tacrolimus therapy as an alternative to thiopurines for maintaining remission in patients with refractory ulcerative colitis.. Journal of clinical gastroenterology 2011, 45(6): 526-530

\section{ISSUE DATE:}

2011-07

URL:

http://hdl.handle.net/2433/197327

\section{RIGHT:}

(c) 2011Lippincott Williams \& Wilkins. This is a non-final version of an article published in final form in Journal of Clinical Gastroenterology, 45(6), July 2011, p 526-530; この論文は 出版社版でありません。引用の際には出版社版をご確認ご利用ください。; This is not the published version. Please cite only the published version. 
Title: Tacrolimus therapy as an alternative to thiopurines for maintaining remission in patients with refractory ulcerative colitis.

Running little: Tacrolimus maintenance therapy for UC

Shuji Yamamoto, $\mathrm{MD}^{1,2}$, Hiroshi Nakase, $\mathrm{MD}, \mathrm{PhD}^{1}$, Minoru Matsuura, $\mathrm{MD}, \mathrm{PhD}^{1}$, Satohiro Masuda, $\mathrm{PhD}^{3}$, Ken-ichi Inui, $\mathrm{PhD}^{4}$, and Tsutomu Chiba, $\mathrm{MD}, \mathrm{PhD}^{1}$.

${ }^{1}$ Department of Gastroenterology and Hepatology, Graduate School of Medicine, Kyoto University, Kyoto, Japan

${ }^{2}$ Research Fellow of the Japan Society for the Promotion of Science, Tokyo, Japan

${ }^{3}$ Department of Pharmacy, Kyoto University Hospital, Kyoto, Japan

${ }^{4}$ President of Kyoto Pharmaceutical University, Kyoto, Japan

\section{Corresponding to:}

Hiroshi Nakase, MD, PhD, Lecturer

Department of Gastroenterology and Hepatology

Graduate School of Medicine, Kyoto University

54 Shogoin Kawahara-cho, Sakyo-ku, Kyoto, 606-8507, Japan

Tel: +81-75-751-4319

Fax: +81-75-751-4303

E-mail: hiropy_n@kuhp.kyoto-u.ac.jp

Conflict of Interest Disclosure: No conflicts of interest exist. 
Acknowledgement: This work was supported by a Grant-in-Aid for Scientific Research (C) from the Ministry of Culture and Science of Japan (grant 18590677); the Kato Memorial Trust for Nambyo Research; the Japan Foundation for Applied Enzymology; the Shimizu Foundation for the Promotion of Immunology Research (to HN); by Grants-in-Aid for Scientific Research (16017240, 16017249, 17013051, 17659212, and 18012029) from the Ministry of Education, Culture, Sports, Science, and Technology of Japan; Grants-in-Aid for Scientific Research (15209024 and 18209027) from JSPS; and a Grant-in-Aid for Research on Measures for Intractable Disease, and Research on Advanced Medical Technology (nano005) from the Ministry of Health, Labor, and Welfare, Japan (to TC). 


\section{Abstract}

Background: Although the efficacy of tacrolimus for inducing remission of refractory ulcerative colitis (UC) is established, its efficacy for maintaining remission of UC has not been evaluated.

Aim: The aim of this study was to evaluate the efficacy of tacrolimus compared with thiopurines for maintaining remission in patients with refractory UC.

Methods: 24 UC patients treated with tacrolimus and 34 treated with thiopurines to maintain remission were enrolled as the tacrolimus group and the thiopurine group, respectively. In the tacrolimus group, $82.8 \%$ of the patients were treated with tacrolimus for induction of the remission, while $70 \%$ of the patients in the thiopurine group were induced remission with either corticosteroid or cytaheresis. Proportions of patients who kept steroid-free remission between the tacrolimus group and the thiopurine group were compared. Maintenance of remission using tacrolimus or thiopurines was defined as no need for other therapies other than aminosalicylates without relapse for at least 3 months. Secondary, to determine whether the response to thiopurines affects the long-term efficacy of tacrolimus maintenance therapy, the overall cumulative relapse-free survival based on the Kaplan-Meier method was estimated in thiopurine-naive or thiopurine-intolerant patients and thiopurine-refractory ones in the tacrolimus group.

Results: Remission was successfully maintained in 17 patients (70.8\%) of the tacrolimus group, and 28 patients (82.4\%) of the thiopurine group. The overall cumulative relapse-free survival of thiopurine-naive or thiopurine-intolerant patients in the tacrolimus group was similar to that in the thiopurine group, and significantly higher than that of thiopurine-refractory patients in the tacrolimus group. 
Conclusions: Maintenance therapy with tacrolimus for patients with UC could be considered an alternative to thiopurine therapy.

Key words: ulcerative colitis, tacrolimus, thiopurines, remission maintenance therapy 


\section{Introduction}

Ulcerative colitis (UC) is an idiopathic, chronic, and inflammatory disorder characterized by diarrhea, rectal bleeding, abdominal pain, fever, anemia, and body weight loss. ${ }^{1}$ Corticosteroid (CS) therapy is used for patients with UC who do not respond to aminosalicylates or those with a severe attack. ${ }^{1,2}$ Although most patients with UC initially respond to CS, approximately $20 \%$ of patients with UC become steroid-dependent within 1 year after initiating CS therapy. ${ }^{3}$ CS is not used as maintenance therapy for patients with UC due to undesirable side effects such as opportunistic infections, diabetes mellitus, osteoporosis, etc. ${ }^{4}$ Therefore, steroid-free remission is an important issue for patients with UC.

Tacrolimus is effective for patients with UC refractory to or dependent on CS, and is usually used as a rescue and bridging therapy before initiating azathioprine (AZA) or 6-mercaptopurine (6-MP) therapy. ${ }^{5-11}$ Several studies have reported the long-term outcomes after tacrolimus therapy for the induction of remission ${ }^{6-9}$; in all of these case series, however, patients received maintenance therapy with other agents, including thiopurines, and infliximab after the induction of clinical remission. To our knowledge, except for a couple of case reports, ${ }^{6,12}$ there are no reports on the effect of tacrolimus as maintenance therapy for patients with refractory UC. Here we evaluated the efficacy of tacrolimus therapy for maintaining remission in patients with refractory UC in comparison with thiopurines, currently the most widely used treatment to maintain steroid-free remission in patients with refractory UC. ${ }^{1,2}$ 


\section{Patients and Methods}

\section{Patients}

Between April 2001 and October 2009, 42 patients with UC who were resistant to or could not be treated with conventional therapy were treated with tacrolimus and 29 (69.0\%) achieved clinical remission. Of those, 23 patients received tacrolimus subsequently for the maintenance of remission. In addition, one patient who failed tacrolimus therapy for inducing remission was maintained remission with tacrolimus after the induction of remission by inflixmab These 24 patients with UC who were treated with tacrolimus to maintain remission after achieving remission were enrolled in this retrospective, observational, single-center study (tacrolimus group). To compare the effect of tacrolimus for maintaining remission of UC, 34 patients who were administered thiopurines to maintain remission during the same period were enrolled as the thiopurine group.

In addition, tacrolimus group was divided into two subgroups according to the response to thiopurines; i.e., thiopurine-refractory tacrolimus group (nine patients did not have a clinical response to thiopurines or relapsed during thiopurine therapy prior to tacrolimus administration) and thiopurine-naive or thiopurine-intolerant tacrolimus group (three patients discontinued thiopurine treatment because of adverse effects and 12 patients had not received thiopurines before tacrolimus therapy). Flow chart of the patients in different groups and subgroups was shown in Figure 1.

This study was reviewed and approved by the Institutional Review Board of Kyoto University. Patients were informed about the potential risks and benefits of tacrolimus therapy and provided written consent to its use. In all cases, the diagnosis was established according to standardized criteria by prior clinical assessment, radiology, 
endoscopy, and histology.

\section{Definition of Response}

Disease activity was measured using a modified Truelove Witts severity index $\left(\right.$ MTWSI) ${ }^{13}$; details are shown in Table 1 . Clinical remission was retrospectively defined as an estimated MTWSI score 4 or less. Relapse was defined as an increase in the MTWSI score to 5 or higher with additional therapies required. To remove the influence of other drugs as much as possible when comparing the efficacy of tacrolimus and thiopurines for steroid-free remission, maintenance of remission was defined as no need for concomitant treatment other than aminosalicylates and topical steroid therapy in addition to tacrolimus or thiopurines without relapse for at least 3 months.

Patients were classified as steroid-resistant or steroid-dependent in accordance with the previously published definition of Ogata et al. ${ }^{5}$

\section{Treatment}

Tacrolimus was administered in its oral formulation. Dosage was adjusted to produce trough tacrolimus whole-blood levels of 10 to $15 \mathrm{ng} / \mathrm{ml}$ to induce remission. After inducing clinical remission, tacrolimus whole-blood trough concentrations were maintained at a lower level, between 5 and $10 \mathrm{ng} / \mathrm{ml} .{ }^{9}$ The initial dose of tacrolimus was $0.1 \mathrm{mg} / \mathrm{kg}$ body weight/day. The mean doses of tacrolimus for inducing and maintaining remission were $7.7 \mathrm{mg} /$ day (range: $2.0-12.0 \mathrm{mg} /$ day) and $5.7 \mathrm{mg} /$ day (range: 2.0-10.0 mg/day), respectively.

For thiopurine maintenance therapy, the dose of AZA or 6-MP was adapted to achieve white blood cell counts between 3000 and 5000 per microliter or 6-thioguanine nucleotide concentrations between 250 and 500 pmol/8 x $10^{8}$ red blood cells. Twenty-four and 10 patients were treated with AZA and 6-MP, respectively. The mean 
initial doses of AZA and 6-MP were 30.5mg/day (range: 25-100 mg/day) and 13.3mg/day (range: 5-30 mg/day), respectively. The mean doses of AZA and 6-MP for the remission phase were $53.0 \mathrm{mg} /$ day (range: $25-100 \mathrm{mg} /$ day) and $25.0 \mathrm{mg} /$ day (range: 5-40 mg/day), respectively.

\section{Assessment and statistics}

The primary endpoint of this study was the proportion of patients in whom remission was successfully maintained. Secondary endpoints included relapse-free survival and treatment safety.

Proportions between groups were compared by Fisher's exact test and continuous variables were compared by Mann-Whitney U-test. Relapse-free survival was assessed using the Kaplan-Meier method. Relapse-free survival of patients who could not maintain remission without concomitant therapies other than aminosalicylates and topical steroid therapy was set as 0 month. A p-value of less than 0.05 was considered to be statistically significant. 


\section{Results}

\section{Patient characteristics}

Baseline characteristics of the patients in each group and subgroup of the tacrolimus group are shown in Table 2 and Table 3, respectively. Except for treatment duration, these baseline characteristics of the tacrolimus group were similar to those of thiopurine group (Table 2).

All patients in the tacrolimus group were treated with aminosalicylates. Four patients (16.7\%) were steroid resistant, 17 patients (70.8\%) were steroid dependent, and the remaining 3 patients (12.5\%) had not been treated with CS. Two patients had received infliximab for the induction of remission. No other patients had been treated with biologics before tacrolimus maintenance therapy.

Therapies for the induction of remission that preceded remission maintenance therapy are summarized in Table 4. In tacrolimus group, three patients received two courses of tacrolimus maintenance therapy and one patient received three treatment courses, and in thiopurine group, six patients received two courses of thiopurine maintenance therapy. Therefore, total 29 and 40 remission induction therapies were performed prior to remission maintenance therapy in tacrolimus and thiopurine group, respectively.

\section{Maintenance of remission}

In tacrolimus group, remission ratio at 3 and 6 months was $70.8 \%$ (17 of 24 patients) and $54.2 \%$ (13 of 24 patients), respectively. In thiopurine group, remission ratio at 3 and 6 months was $82.4 \%$ (28 of 34 patients) and 73.5\% (25 of 34 patients), respectively. Proportions of patients who were stayed in remission at 3 and 6 months were not statistically different between the two groups ( $p=0.3494$ and 0.1647 , respectively; Fisher's exact test). The mean durations of steroid-free remission in the tacrolimus 
group and the thiopurine group were 9.2 months (range: 0-50.7 months) and 14.0 months (range: 0-51.6 months), respectively. There was no statistical difference between the two groups again ( $p=0.1114$; Mann-Whitney U-test).

Although there was no significant difference between the two groups, the duration of remission in the tacrolimus group tended to be shorter than that in thiopurine group. Therefore, to determine whether the response to thiopurine therapy affects the long-term efficacy of tacrolimus maintenance therapy, the overall cumulative relapse-free survival based on the Kaplan-Meier method was estimated in the thiopurine-naive or thiopurine-intolerant tacrolimus group and the thiopurine-refractory tacrolimus group. Relapse-free survival in patients in the thiopurine-naive or thiopurine-intolerant tacrolimus group was comparable to that in the thiopurine group ( $p=0.5594$; log-rank test) and was significantly higher than that in the thiopurine-refractory tacrolimus group ( $p=0.0104$; log-rank test; Figure 2.)

\section{Adverse Effects}

The frequency of adverse events during tacrolimus therapy is shown in Table 5. Tacrolimus withdrawal was necessary in 4 patients (16.7\%). 1 patient (4.2\%) contracted bacterial pneumonia. He had received a combination of tacrolimus and AZA and recovered with antibiotic administration and discontinuation of the tacrolimus and AZA. A temporary rise in serum creatinine levels above $1.3 \mathrm{mg} / \mathrm{dl}$ occurred in 4 patients (16.7\%). In 3 cases, tacrolimus withdrawal was necessary and serum creatinine levels normalized after discontinuation. In the other case, renal function normalized with dose reduction of tacrolimus.

The thiopurines were discontinued due to side effects in 5 (14.7\%) of 34 patients undergoing thiopurine maintenance therapy. Of those, 1 patient $(2.9 \%)$ contracted 
bacterial pneumonia as described above, 1 patient (2.9\%) developed leukopenia, 1 patient (2.9\%) developed pancreatitis, and 2 patients (5.9\%) experienced nausea. Herpes progenitalis $(2.9 \%, n=1)$ and mild leukopenias $(5.9 \%, n=2)$ were also observed. All of the patients in both groups recovered with conventional therapy. There was no mortality in either of the groups. 


\section{Discussion}

The findings of the present study demonstrated that the effects of tacrolimus as maintenance therapy in thiopurine-naive or thiopurine-intolerant patients with UC are comparable to those of thiopurines. To our knowledge, this is the first study to show that tacrolimus therapy is valuable for maintaining remission in patients with refractory UC in comparison with thiopurine therapy.

First, we investigated the efficacy of thiopurines for maintaining remission in patients with refractory UC enrolled in this study. Our study showed that the proportions of UC patients maintaining steroid-free remission with thiopurines at 1 year and 3 years were $59.2 \%$ and $36.5 \%$, respectively (Figure 2). In a prospective, observational cohort study by Chebli et al., ${ }^{14}$ the proportion of patients with steroid-dependent UC who received AZA for 3 years and remained in steroid-free remission was $45 \%$ and $57.5 \%$ on an intention-to-treat basis and per protocol basis, respectively. Fraser et al. ${ }^{15}$ reviewed the clinical notes of 622 patients with inflammatory bowel disease (IBD; 272 Crohn's disease, 346 UC, and 4 indeterminate colitis) who were treated with AZA to maintain remission and demonstrated that the relapse-free rate based on a Cox regression analysis was $63 \%$ at 60 months. Although the relapse-free rate in the later study seems to be higher than that in thiopurine therapy in a previous study and in the present study, patients who received AZA for less than 3 months were excluded in the study by Fraser et al., whereas all patients who were administered thiopurines were enrolled in other studies. Thus, in our study, the clinical outcome of patients treated with thiopurines was similar to that in previous reports. ${ }^{14,15}$

Then, we investigated the efficacy of tacrolimus as maintenance therapy for patients with refractory UC in comparison with thiopurines. Our study demonstrated that the 
proportions of patients intolerant or naive to thiopurines who could maintaine steroid-free remission with tacrolimus at 1 year and 3 years were 51.1\% and 19.2\%, respectively (Figure 2), which was similar to that in patients with thiopurines. In contrast, the proportions of patients that were refractory to thiopurines at 1 year and 3 years were $25.0 \%$ and $0 \%$, respectively (Figure 2). Relapse-free survival in this group was significantly lower than that in thiopurine group. These data suggested that administration of tacrolimus with trough levels of 5 to $10 \mathrm{ng} / \mathrm{ml}$ as maintenance therapy could be an alternative therapy for UC patients intolerant to thiopurines, but might be less effective in thiopurine-refractory patients with UC.

Some reports recommend tacrolimus trough levels of 5 to $10 \mathrm{ng} / \mathrm{ml}$ for long-term administration to avoid rejection in patients with liver, renal, and small bowel transplantation. ${ }^{16,17}$ Our previous report also showed that the same trough range might be optimal for maintaining remission in patients with refractory UC based on its effect and safety. ${ }^{9}$ According to these previous reports, in the study we treated UC patients by adjusting the tacrolimus trough levels to 5 to $10 \mathrm{ng} / \mathrm{ml}$ in this study. Our data suggested, however, that patients with UC who are refractory to thiopurines should be controlled with higher trough levels of tacrolimus, a combination of thiopurines and tacrolimus, or infliximab.

One limitation of this study concerns the difference of the induction therapy between the tacrolimus group and the thiopurine group. In fact, $82.8 \%$ of the patients in the tacrolimus group were treated with tacrolimus, while $70 \%$ of the patients in the thiopurine group received either CS or cytaheresis (Table 4). Therefore, we could not exclude the possibility that patients in the thiopurine group had less severe disease than those in the tacrolimus group. To solve this issue, additional studies might be required 
with enrolling patients who received the same induction therapies.

Finally, we evaluated the adverse effects related to long-term administration of tacrolimus therapy. In this study, tremor was the most frequent side effect and severe adverse events rarely occurred during tacrolimus maintenance therapy. The frequency of drug withdrawal due to side effects was similar between the tacrolimus and thiopurine groups, indicating that long-term tacrolimus administration with trough levels of 5 to $10 \mathrm{ng} / \mathrm{ml}$ could be tolerable in patients with refractory UC.

In conclusion, our study demonstrated that tacrolimus therapy is a viable alternative for maintaining steroid-free remission in UC patients intolerant to thiopurines, although its efficacy and the therapeutic strategy for thiopurine-refractory UC remain to be established. 


\section{Figure legend}

Figure 1. A flow chart of patients in different groups and subgroups.

Figure 2. Relapse-free time intervals of thiopurine-refractory patients and thiopurine-naive or thiopurine-intolerant patients in the tacrolimus group and in the thiopurine group. The overall cumulative relapse-free survival of thiopurine-refractory patients in the tacrolimus group was significantly lower than that in the other two groups (log-rank test; $p=0.0104$ [vs thiopurine-naive or -intolerant patients] and $p=0.0008$ [vs thiopurine group]). There was no statistical difference between the thiopurine-naive or thiopurine-intolerant tacrolimus and thiopurine groups ( $p=0.5594)$. 


\section{References}

1. Baumgart DC, Sandborn WJ. Inflammatory bowel disease: clinical aspects and established and evolving therapies. Lancet 2007;369:1641-1657

2. Sands BE. Inflammatory bowel disease: past, present, and future. J Gastroenterol 2007;42:16-25.

3. Faubion WA Jr, Loftus EV Jr, Harmsen WS, Zinsmeister AR, Sandborn WJ. The natural history of corticosteroid therapy for inflammatory bowel disease: a population-based study. Gastroenterology 2001;121:255-260.

4. Swartz SL, Dluhy RG. Corticosteroids: clinical pharmacology and therapeutic use. Drugs 1978;16:238-255.

5. Ogata $\mathrm{H}$, Matsui $\mathrm{T}$, Nakamura $\mathrm{M}$ et al. A randomized dose finding study of oral tacrolimus (FK506) therapy in refractory ulcerative colitis tacrolimus. Gut 2006;55:1255-1262.

6. Baumgart DC, Pintoffl JP, Sturm A, Wiedenmann B, Dignass AU. Tacrolimus is safe and effective in patients with severe steroid- refractory or steroid-dependent inflammatory bowel disease-A long-term follow-up. Am J Gastroenterol 2006;101:1048-1056.

7. Fellermann K, Tanko Z, Herrlinger KR et al. Response of refractory colitis to intravenous or oral tacrolimus (FK506). Inflamm Bowel Dis 2002;8:317-324. 8. Ng SC, Arebi N, Kamm MA. Medium-term results of oral tacrolimus treatment in refractory inflammatory bowel disease. Inflamm Bowel Dis 2007;13:129-134.

9. Yamamoto S, Nakase H, Mikami S et al. Long-term effect of tacrolimus therapy in patients with refractory ulcerative colitis. Aliment Pharmacol Ther 2008;28:589-597. 10. Högenauer C, Wenzl HH, Hinterleitner TA, Petritsch W. Effect of oral tacrolimus 
(FK 506) on steroid-refractory moderate/severe ulcerative colitis. Aliment Pharmacol Ther 2003;18:415-423.

11. Ziring DA, Wu SS, Mow WS, Martín MG, Mehra M, Ament ME. Oral tacrolimus for steroid-dependent and steroid-resistant ulcerative colitis in children. J Pediatr Gastroenterol Nutr 2007; 45:306-311.

12. Nakase H, Mikami S, Matsuura M et al. Rescue therapy with tacrolimus for a patient with severe ulcerative colitis refractory to combination leukocytapheresis and high-dose corticosteroid therapy. Intern Med 2007;46:717-720.

13. Lichtiger S, Present DH. Preliminary report: cyclosporin in treatment of severe active ulcerative colitis. Lancet 1990;336:16-19.

14. Chebli LA, Chaves LD, Pimentel FF et al. Azathioprine maintains long-term steroid-free remission through 3 years in patients with steroid-dependent ulcerative colitis. Inflamm Bowel Dis 2010;16:613-619.

15. Fraser AG, Orchard TR, Jewell DP. The efficacy of azathioprine for the treatment of inflammatory bowel disease: a 30 year review. Gut. 2002;50:485-489.

16. Langnas AN. Advances in small-intestine transplantation. Transplantation. 2004;15:S75-78.

17. McMaster P, Mirza DF, Ismail T, Vennarecci G, Patapis P, Mayer AD. Therapeutic drug monitoring of tacrolimus in clinical transplantation. Ther Drug Monit 1995;17:602-605. 
Table 1. Modified Truelove Witts severity index (MTWSI) ${ }^{14}$

\begin{tabular}{|c|c|c|c|c|c|c|}
\hline score & 0 & 1 & 2 & 3 & 4 & 5 \\
\hline bowel movement & $0-2$ & $3-4$ & $5-6$ & $7-9$ & $>=10$ & \\
\hline nocturnal diarrhea & no & yes & & & & \\
\hline visible blood in stool & $0 \%$ & $<50 \%$ & $>=50 \%$ & $100 \%$ & & \\
\hline $\begin{array}{l}\text { abdominal } \\
\text { tenderness }\end{array}$ & none & mild & moderate & severe & & \\
\hline $\begin{array}{c}\text { abdominal } \\
\text { pain/cramping }\end{array}$ & none & mild & moderate & severe & & \\
\hline $\begin{array}{c}\text { need for } \\
\text { antidiarrheals }\end{array}$ & no & yes & & & & \\
\hline general status & perfect & $\begin{array}{l}\text { very } \\
\text { good }\end{array}$ & good & average & poor & terrible \\
\hline fecal incontinence & no & yes & & & & \\
\hline
\end{tabular}

Remission category : 4 or less 
Table 2. Patients' baseline characteristics in the tacrolimus group and the thiopurine group

\begin{tabular}{|c|c|c|c|}
\hline & Tacrolimus group & Thiopurine group & $p$-value \\
\hline Age at diagnosis & $23.4^{\mathrm{a}}$ & $29.3^{\mathrm{a}}$ & 0.4334 \\
\hline [median (range)] & $(11.9-74.7)$ & $(15.3-60.8)$ & \\
\hline Age at start of the therapy & $27.5^{\mathrm{a}}$ & $36.2^{\mathrm{a}}$ & 0.3619 \\
\hline [median (range)] & $(15.9-78.1)$ & $(16.8-65.0)$ & \\
\hline Disease duration prior the & $3.6^{\mathrm{b}}$ & $3.6^{\mathrm{b}}$ & 0.7116 \\
\hline therapy [median (range)] & $(0.4-22.8)$ & $(0.8-31.6)$ & \\
\hline Follow up duration & $23.0^{\mathrm{c}}$ & $34.4^{\mathrm{c}}$ & 0.4207 \\
\hline [median (range)] & $(5.1-87.1)$ & $(3.4-86.0)$ & \\
\hline Treatment duration & $13.2^{\mathrm{c}}$ & $25.7^{c}$ & 0.0187 \\
\hline [median (range)] & $(1.8-53.7)$ & $(3.4-61.5)$ & \\
\hline Sex & & & 0.2913 \\
\hline Men (\%) & $12(50 \%)$ & $22(64.7 \%)$ & \\
\hline Women (\%) & 12 (50\%) & $12(35.3 \%)$ & \\
\hline Disease extent & & & 1.0000 \\
\hline Extensive (\%) & 17 (70.8\%) & $24(70.6 \%)$ & \\
\hline Left-sided (\%) & 7 (29.2\%) & $10(29.4 \%)$ & \\
\hline Response to corticosteroids & & & 0.3428 \\
\hline Steroid resistance (\%) & $4(16.7 \%)$ & $5(14.7 \%)$ & \\
\hline Steroid dependence (\%) & 17 (70.8\%) & 28 (82.4\%) & \\
\hline Steroid naive (\%) & 3 (12.5\%) & $1(2.9 \%)$ & \\
\hline
\end{tabular}

${ }^{\mathrm{a}}$ Age, ${ }^{\mathrm{b}}$ years, and ${ }^{\mathrm{c}}$ months are shown.

Numbers of patients are shown unless specified. 
Table 3. Baseline characteristics of patients in each tacrolimus subgroup

\begin{tabular}{|c|c|c|c|}
\hline & $\begin{array}{c}\text { Thiopurine-refeactory } \\
\text { group }\end{array}$ & $\begin{array}{l}\text { Thiopurine-naive or } \\
\text { thiopurine- intolerant group }\end{array}$ & $p$-value \\
\hline Age at diagnosis & $21.7^{\mathrm{a}}$ & $23.8^{\mathrm{a}}$ & 0.4207 \\
\hline [median (range)] & $(15.3-56.0)$ & $(11.9-74.7)$ & \\
\hline Age at start of the therapy & $30.4^{\mathrm{a}}$ & $27.2^{\mathrm{a}}$ & 0.7565 \\
\hline [median (range)] & $(16.5-67.7)$ & $(15.9-78.1)$ & \\
\hline Disease duration prior the & $4.8^{b}$ & $3.4^{\mathrm{b}}$ & 0.0131 \\
\hline therapy [median (range)] & $(1-22.8)$ & $(0.4-4.8)$ & \\
\hline Follow up duration & $12.67^{c}$ & $44.6^{\mathrm{c}}$ & 0.1440 \\
\hline [median (range)] & $(5.1-61.2)$ & $(6.5-87.1)$ & \\
\hline Treatment duration & $12.67^{\mathrm{c}}$ & $15.6^{\mathrm{c}}$ & 0.5711 \\
\hline [median (range)] & $(5.1-44.4)$ & $(1.8-53.7)$ & \\
\hline Sex & & & 0.4003 \\
\hline Men (\%) & $3(33.3 \%)$ & $9(75.0 \%)$ & \\
\hline Women (\%) & $6(66.7 \%)$ & $3(25.0 \%)$ & \\
\hline Disease extent & & & 1.0000 \\
\hline Extensive (\%) & $6(66.7 \%)$ & $11(73.3 \%)$ & \\
\hline Left-sided (\%) & $3(33.3 \%)$ & $4(26.7 \%)$ & \\
\hline Response to corticosteroids & & & 0.8241 \\
\hline Steroid resistance (\%) & $1(11.1 \%)$ & $3(20.0 \%)$ & \\
\hline Steroid dependence (\%) & $7(77.8 \%)$ & $10(66.7 \%)$ & \\
\hline Steroid naive (\%) & $1(11.1 \%)$ & 2 (13.3\%) & \\
\hline
\end{tabular}

${ }^{a}$ Age, ${ }^{b}$ years, and ${ }^{c}$ months are shown. Numbers of patients are shown unless specified. 
Table 4. Therapies for the induction of remission prior to maintenance therapies

\begin{tabular}{lcc}
\hline & Tacrolimus group & Thiopurine group \\
\hline Prednisolone (\%) & $1(3.4 \%)$ & $20(50.0 \%)$ \\
Tacrolimus (\%) & $24(82.8 \%)$ & $11(27.5 \%)$ \\
Infliximab (\%) & $2(6.9 \%)$ & $1(2.5 \%)$ \\
Cytoapheresis (\%) & $2(6.9 \%)$ & $8(20.0 \%)$ \\
\hline
\end{tabular}

Numbers of patients are shown. 
Table 5. Adverse events that developed during tacrolimus treatment

\begin{tabular}{lcc}
\hline Adverse events & Cases & (\%) \\
\hline Tremor & 5 & $(20.8 \%)$ \\
Renal function impairment & 4 & $(16.7 \%)$ \\
${\text { (rise in creatinine above } 1.3 \mathrm{mg} / \mathrm{dl})^{\mathrm{a}}}^{\text {Hot flashes }}$ & & \\
Bacterial pnuemonia & 3 & $(12.5 \%)$ \\
Hyperkalemia & 1 & $(4.2 \%)$ \\
Epigastralgia & 1 & $(4.2 \%)$ \\
Headache & 1 & $(4.2 \%)$ \\
\hline
\end{tabular}

${ }^{\mathrm{a}}$ In one case, tacrolimus withdrawal was not necessary. 


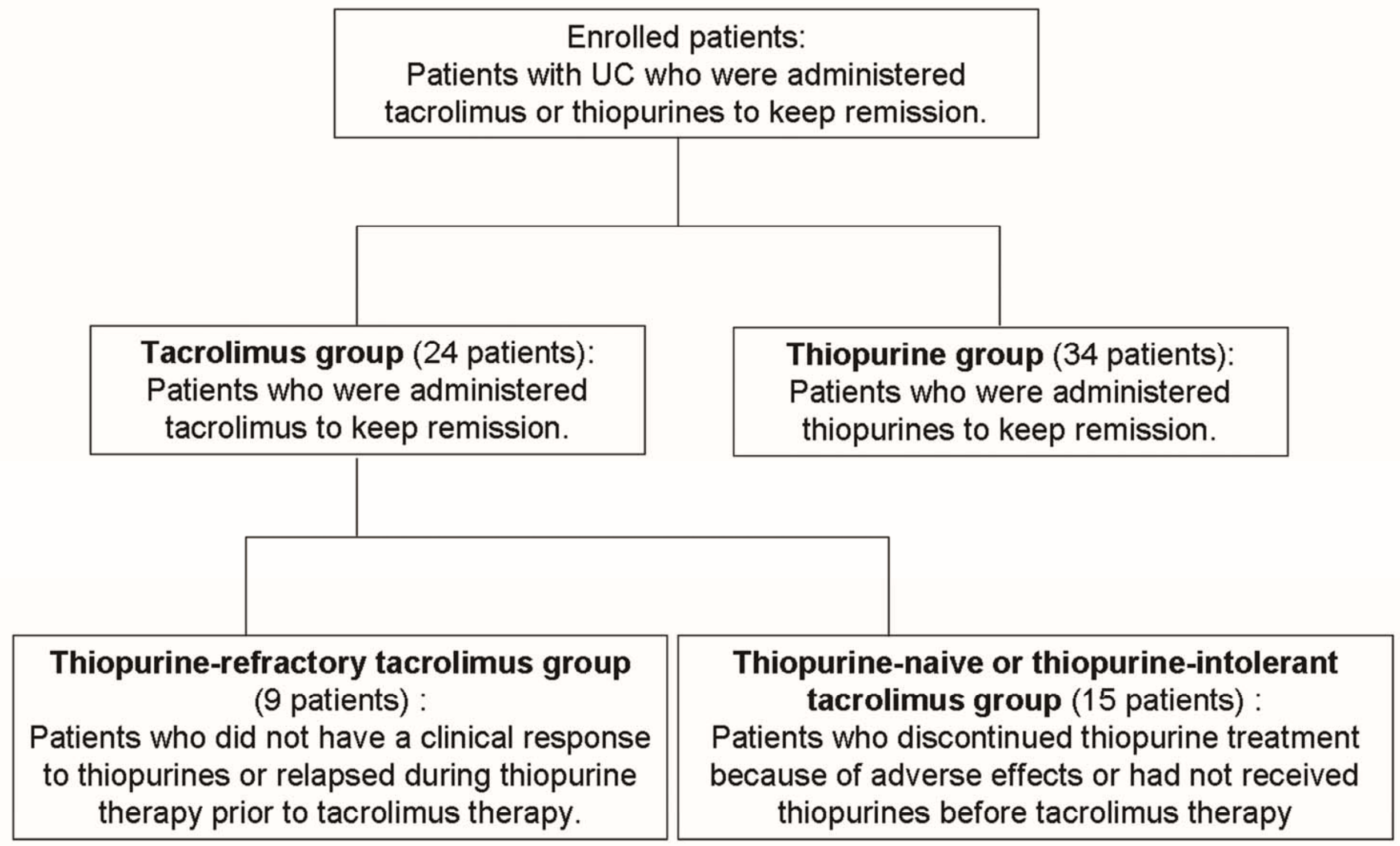

Figure 1 


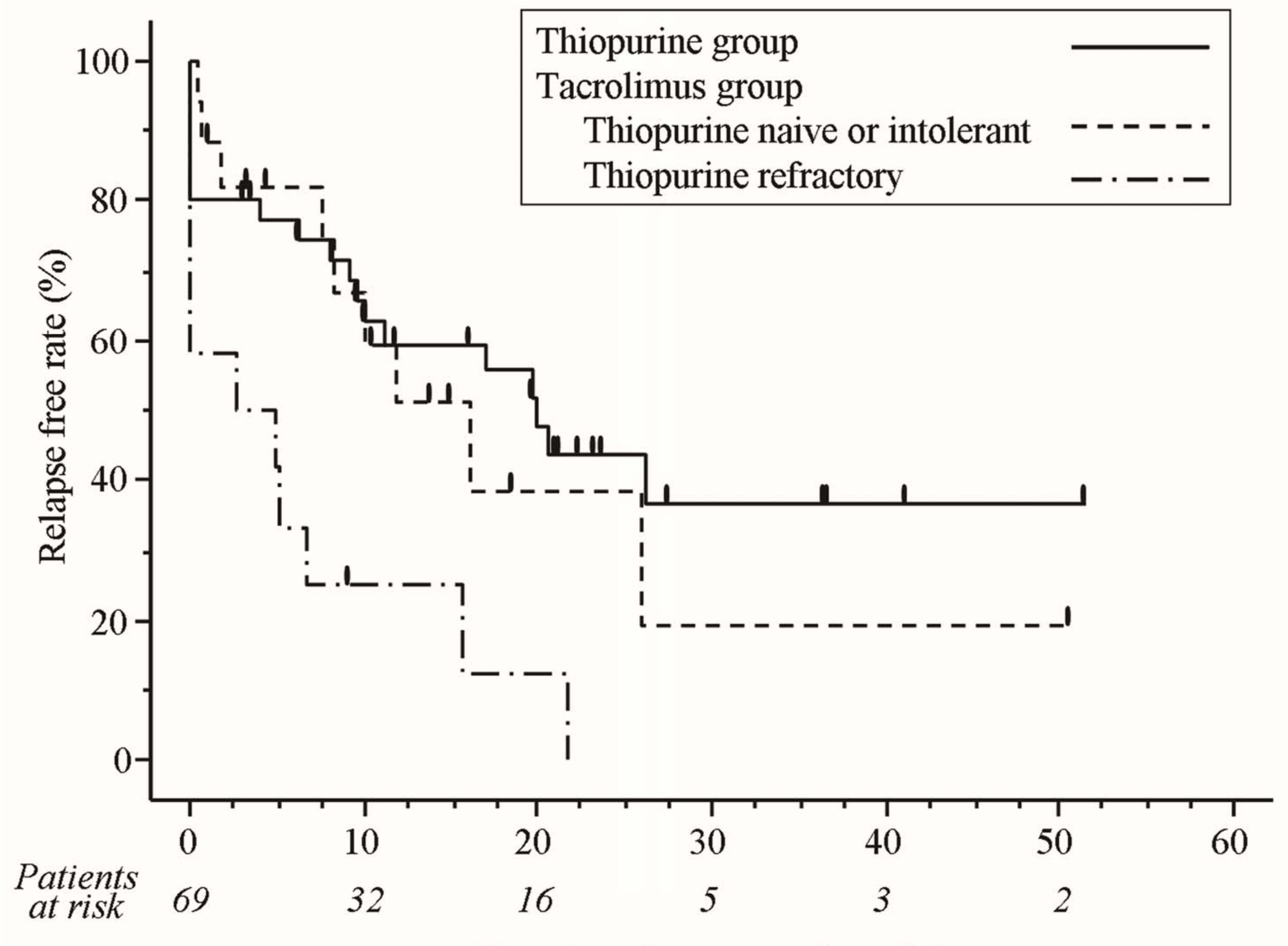

Duration of treatment (months)

Figure 2 